International Journal of Bifurcation and Chaos

(c) World Scientific Publishing Company

\title{
Mean Exit Time and Escape Probability for a Tumor Growth System under Non-Gaussian Noise*
}

\author{
Jian Ren \\ School of Mathematics and Statistics, Huazhong University of Sciences and Technology \\ Wuhan 430074, China \\ renjian0371@gmail.com \\ Chujin Li \\ School of Mathematics and Statistics, Huazhong University of Sciences and Technology \\ Wuhan 430074, China \\ lichujin@mail.hust.edu.cn \\ Ting Gao \\ Department of Applied Mathematics, Illinois Institute of Technology \\ Chicago, IL 60616, USA \\ Xingye Kan \\ Department of Applied Mathematics, Illinois Institute of Technology \\ Chicago, IL 60616, USA \\ Jinqiao Duan \\ Department of Applied Mathematics, Illinois Institute of Technology \\ Chicago, IL 60616, USA \\ duan@iit.edu \\ Received (to be inserted by publisher)
}

\begin{abstract}
Effects of non-Gaussian $\alpha$-stable Lévy noise on the Gompertz tumor growth model are quantified by considering the mean exit time and escape probability of the cancer cell density from inside a safe or benign domain. The mean exit time and escape probability problems are formulated in a differential-integral equation with a fractional Laplacian operator. Numerical simulations are conducted to evaluate how the mean exit time and escape probability vary or bifurcates when $\alpha$ changes. Some bifurcation phenomena are observed and their impacts are discussed.

Keywords: Fractional Laplacian operator; bifurcation; tumor growth model; non-Gaussian noise; $\alpha$-stable Lévy motion; quantifying uncertainty
\end{abstract}

\section{Introduction}

Mathematical models have been proposed to describe the evolution of numerous biological phenomena such as cancer growth. The deterministic Gompertz growth model for a tumor density growth is given by the

*This research was partly supported by the NSF Grants 0731201 and 1025422, the NSFC grants 10971225 , 11028102 and 10901065, and the Fundamental Research Funds for the Central Universities, HUST 2010ZD037. 
following differential equation

$$
\frac{d x}{d t}=(A-B \ln x) x
$$

where $x(t)$ describes the density of cancer cells at time $t$, parameters $A$ and $B$ denote growth and decay rates respectively.

This is a deterministic model, nevertheless. As there is a discrepancy between clinical data and basic theory due to subtle environmental fluctuations, we need a stochastic part to describe the fluctuation. A stochastic model proposed as a functional Fokker-Planck equation in consideration of both fission and mortality has been considered in Lo, 2007] and a diffusion process with modified infinitesimal mean has been studied in [Gutierrez-jaimez et al., 2007]. In [Lo, 2009] and Albano \& Giorno, 2009] a multiplicative noise of Brownian motion has been considered and in [Albano \& Giorno, 2006] a deterministic parameter has been modified by a group of random variables to depict the stochastic fluctuation. [Lo, 2009] compared the effect of different therapies by giving their probability density functions(pdfs) and Albano \& Giorno, 2009], Albano \& Giorno, 2006] both deal with the first exit time problem.

In the present paper we consider the Gompertz growth model driven by the non-Gaussian $\alpha$-stable noise with jumps and quantify the dynamics in terms of mean exit time and escape probability. Specifically, we consider the evolution of the tumor growth in the density range that cannot be diagnosed, and compute the likelihood and mean time for a tumor to become diagnosable or malignant. We examine how this depends on $\alpha$ values. To this end, we investigate the differential equation driven by symmetric $\alpha$-stable Lévy process $L_{t}^{\alpha}$ with characteristics $(0,1, \nu)$ with jump measure $\nu(d y)=d y /|y|^{1+\alpha}$

$$
d X=[(A-B \ln X) X] d t+d L_{t}^{\alpha}, \quad X(0)=x .
$$

where $x$ is the initial tumor density.

The paper is arranged as follows. We present a brief introduction about Lévy process in section 2 and then a stochastic tumor growth model in section 3. Numerical experiments are conducted in section 4 .

\section{Lévy process}

A Lévy process $\left(L_{t}, t \geq 0\right)$ is a stochastic process defined on a probability space $(\Omega, \mathcal{F}, P)$ with the following properties:

(i) $L_{0}=0$ (a.s.);

(ii) $L_{t}$ has independent and stationary increments, i.e. for each $n$ and $0 \leq t_{1} \leq t_{2} \leq \cdots \leq t_{n+1}<\infty$ the random variables $\left(L_{t_{j+1}}-L_{t_{j}}, 1 \leq j \leq n\right)$ are independent and each $L_{t_{j+1}}-L_{t_{j}}$ and $L_{t_{j+1}-t_{j}}$ are equal in law both satisfy a non-Gaussian distribution.

(iii) $L_{t}$ has stochastically continuous, i.e. for all $c>0, s>0$

$$
\lim _{t \rightarrow s} P\left(\left|L_{t}-L_{s}\right|>c\right)=0
$$

(iv) The paths of $L_{t}$ are $\mathbb{P}$-almost surely right continuous with left limits.

For the characteristic exponent $\Psi_{t}(\lambda)=\log \mathbb{E}\left(e^{i \lambda L_{t}}\right)$, with the fact that $L_{t}$ has independent and stationary increment we get $\mathbb{E}\left(e^{i \lambda L_{t}}\right)=e^{t \Psi_{1}(\lambda)}=: e^{t \Psi(\lambda)}$, therefore in the next paragraph we refer to $\Psi(\lambda)$ as a characteristic exponent of the Lévy process.

Lévy-Khintchine formula [Applebaum, 2009; Kyprianou, 2006] for Lévy process: Suppose that $a \in \mathbb{R}, \sigma \geq 0$ and $\nu$ is a measure concentrated on $\mathbb{R} \backslash\{0\}$ such that $\int_{\mathbb{R}}\left(1 \wedge x^{2}\right) \mu(d x)<\infty$. From this triple $(a, \sigma, \nu)$ defined for each $\lambda \in \mathbb{R}$,

$$
\Psi(\lambda)=i a \lambda-\frac{1}{2} \sigma^{2} \lambda^{2}-\int_{\mathbb{R}}\left(1-e^{i \lambda x}+i \lambda x I_{|x|<1}\right) \nu(d x)
$$

Then there exits a probability space $(\Omega, \mathcal{F}, \mathbb{P})$ on which a Lévy process is defined having characteristic exponent $\Psi$. 
It can be seen that after some reorganization the characteristic exponent of Lévy process can be rewritten as

$$
\begin{aligned}
\Psi(\lambda)= & \left\{i a \lambda-\frac{1}{2} \sigma^{2} \lambda^{2}\right\} \\
& -\left\{\nu(\mathbb{R} \backslash(-1,1)) \int_{|x| \geq 1}\left(1-e^{i \lambda x}\right) \frac{\nu(d x)}{\nu(\mathbb{R} \backslash(-1,1))}\right\} \\
& -\left\{\int_{0<|x|<1}\left(1-e^{i \lambda x}+i \lambda x\right) \nu(d x)\right\} \\
=: & \Psi^{(1)}-\Psi^{(2)}-\Psi^{(3)}
\end{aligned}
$$

where $\Psi^{(1)}, \Psi^{(2)}$ are respectively the characteristic exponent of $X_{t}^{(1)}=-\sigma B_{t}+a t, \quad t \geq 0$ and $X_{t}^{(2)}=$ $\sum_{i=1}^{N_{t}} \xi_{i}, \quad t \geq 0$, here $\left\{N_{t}: t \geq 0\right\}$ is a Poisson process with rate $\nu(\mathbb{R} \backslash(-1,1))$ and $\left\{\xi_{i}: t \geq 0\right\}$ are i.i.d with distribution $\nu(d x) / \nu(\mathbb{R} \backslash(-1,1))$ concentrated on $\{x:|x| \geq 1\}$ (if $\nu(\mathbb{R} \backslash(-1,1))=0$ process $X_{t}^{(2)}$ is identically zero).

We have a result from [Kyprianou, 2006] to say the composition of a Lévy process.

Lévy-Itô decomposition: Given any $a \in \mathbb{R}, \sigma \geq 0$ and measure $\nu$ concentrated on $\mathbb{R} \backslash\{0\}$ satisfying $\int_{\mathbb{R} \backslash\{0\}}\left(|x|^{2} \wedge 1\right) \nu(d x)<\infty$, there exists a probability space on which three independent Lévy processes $X^{(1)}, X^{(2)}, X^{(3)}$ exit, where $X^{(1)}$ is a linear Brownian motion with drift with characteristic exponent $\Psi^{(1)}$, $X^{(2)}$ is a compound Poisson process with characteristic exponent $\Psi^{(2)}$ and $X^{(3)}$ is a square integrable martingale with an almost surely countable number of jumps on each finite time interval which are of magnitude less than unity and with characteristic exponent $\Psi^{(3)}$. By taking $X=X^{(1)}-X^{(2)}-X^{(3)}$ we have that there exist a probability space on which a Lévy process is defined with characteristic exponent

$$
\Psi(\lambda)=i a \lambda-\frac{1}{2} \sigma^{2} \lambda^{2}-\int_{\mathbb{R}}\left(1-e^{i \lambda x}+i \lambda x I_{(|x|<1)}\right) \mu(d x)
$$

for $\lambda \in \mathbb{R}$.

A random variable $X$ is said to be stable if for all $n>0$, the distribution of $X$ and $S_{n}=\frac{X_{1}+\cdots+X_{n}-a_{n}}{\sigma_{n}}$ are equivalent, where $X_{1}, \cdots, X_{n}$ are a sequence of i.i.d random variable, $a_{n} \in \mathbb{R}$ and $\sigma_{n}>0$ for $n \in \mathbb{N}$. It is well known from [Applebaum, 2009; Feller, 1971] that the only possible choice is $\sigma_{n}=n^{1 / \alpha}$ for $\alpha \in(0,2]$ so we refer to $\alpha$ as the index.

For $\alpha \in(0,1) \cup(1,2)$ stable variables have characteristic exponents of the form

$$
\Psi(\lambda)=i a \lambda-c^{\alpha}|\lambda|^{\alpha}\left(1-i \beta \tan \frac{\pi \alpha}{2} \operatorname{sgn} \lambda\right),
$$

where $\beta \in[-1,1], a \in \mathbb{R}, c>0$ and the sign function $\operatorname{sgn} \lambda=I_{(\lambda>0)}-I_{(\lambda<0)}$;

for $\alpha=1$, the characteristic exponents have the form

$$
\Psi(\lambda)=i a \lambda-c|\lambda|\left(1+i \beta \frac{2}{\pi} \operatorname{sgn} \lambda \log |\lambda|\right),
$$

where $\beta \in[-1,1], a \in \mathbb{R}$ and $c>0$;

for the special case $\alpha=2$,

$$
\Psi(\lambda)=i a \lambda-\frac{1}{2} \sigma^{2} \lambda^{2}
$$

and the measure

$$
\nu(d x)=\left\{\begin{array}{lll}
\frac{c_{1}}{x^{1+\alpha}} d x & \text { for } & x \in(0, \infty) ; \\
\frac{c_{2}}{(-x)^{1+\alpha}} d x & \text { for } \quad x \in(-\infty, 0),
\end{array}\right.
$$

where $c=c_{1}+c_{2}, c_{1}, c_{2} \geq 0$ and $\beta=\frac{c_{1}-c_{2}}{c_{1}+c_{2}}$ if $\alpha \in(0,1) \cup(1,2)$. The case $\beta=0$, i.e. $c_{1}=c_{2}$, is called symmetric $\alpha$-stable Lévy process. 
The (infinitesimal) generator $A$ of a Lévy process $X(t)$ is defined as $A \varphi=\lim _{t \downarrow 0} \frac{T_{t} \varphi-\varphi}{t}$ where $\left(T_{t} \varphi\right)(x)=$ $\mathbb{E}_{x}\left(\varphi(X(t))\right.$ for any $\varphi \in D_{A}$ (the domain of generator $A$ ). It has the expression [Applebaum, 2009]

$$
(A \varphi)(x)=a \varphi^{\prime}(x)+\frac{1}{2} \sigma^{2} \varphi^{\prime \prime}(x)+\int_{\mathbb{R} \backslash\{0\}}\left[\varphi(x+y)-\varphi(x)-y \varphi^{\prime}(x) I_{|y|<1}\right] \nu(d y) .
$$

\section{Tumor growth under $\alpha$-stable Lévy noise}

In this section we consider the stochastic model driven by symmetric $\alpha$-stable Lévy process, i.e. a Lévy process $L_{t}$, in which each $L_{t}$ is a stable random variable and here for simplicity we take $c_{1}=c_{2}=1$. We refer to the monoclonal benign parathyroid tumor and take $A=6.46$ year $^{-1}, B=0.314$ year $^{-1}$, corresponding to a parathyroid tumor mean age of 19.6 years. Here we investigate in the range of the tumor density that cannot be diagnosed, we take the two boundary points $S_{1}=1$ and $S_{2}=1.074 \times 10^{8}$ (the tumor cell density $x=1.074 \times 10^{8}$ is the smallest diagnoseable mass), how the tumor density progresses. When a tumor's density is bigger than $1.074 \times 10^{8}$, we say it becomes malignant, while if its density is smaller than 1 , it is not diagnosable.

Based on the deterministic Gompertz model, the corresponding stochastic model is given by

$$
d X=[(A-B \ln X) X] d t+d L_{t}^{\alpha}, \quad X(0)=x,
$$

where $L_{t}^{\alpha}$ is with the characteristics $(0,1, \nu)$. Here the jump measure $\nu(d y)=d y /|y|^{1+\alpha}$. It is known Applebaum, 2009] that the infinitesimal generator $A$ for $L_{t}^{\alpha}$ is

$$
(A f)(x)=\frac{1}{2} f^{\prime \prime}(x)+\int_{\mathbb{R} \backslash\{0\}} \frac{f(x+y)-f(x)-y f^{\prime}(x) I_{|y|<1}}{|y|^{1+\alpha}} d y .
$$

Due to the Lévy-Itô decomposition the generator for the process $X(t)$ in (3) is then

$$
A f=(A-B \ln x) x f^{\prime}(x)+\frac{1}{2} f^{\prime \prime}(x)+\int_{\mathbb{R} \backslash\{0\}} \frac{f(x+y)-f(x)-y f^{\prime}(x) I_{|y|<1}}{|y|^{1+\alpha}} d y .
$$

In this symmetric $\alpha$-stable Lévy motion case, the integral operator is related to the fractional Laplacian operator Albeverrio, Rudiger \& Wu, 2000; Guan \& Ma, 2005; Chen, Kim \& Song, 2010; Caffarelli \& Silvestre, 2007]. Namely, for $\alpha \in(0,2)$

$$
\int_{\mathbb{R} \backslash\{0\}}[u(x+y)-u(x)] \nu_{\alpha}(d y)=c_{\alpha}(-\Delta)^{\frac{\alpha}{2}} u(x),
$$

where

$$
c_{\alpha} \triangleq \int_{\mathbb{R} \backslash\{0\}}(\cos y-1) \nu_{\alpha}(d y)<0 .
$$

Note that when $\alpha=2, L_{2}=\Delta$.

We consider two issues: Mean exit time and escape probability. We quantify the stochastic dynamics using these two tools. Especially, we examine possible bifurcation phenomena when the stability parameter $\alpha$ various in $(0,2)$.

The mean exit time

$$
u(x)=\mathbb{E} \inf \left\{t \geq 0, X_{t}(\omega, x) \notin D\right\},
$$

from a bounded interval $D$, is the first time that the tumor density $X(t)$, initially at value $x$ in $D$, gets out of $D$. The mean exit time $u(x)$, from the diagnoseable range $D=\left(1,1.074 \times 10^{8}\right)$, satisfies an integraldifferential equation Brannan, Duan \& Ervin, 1999]

$$
\begin{aligned}
A u(x) & =-1, \quad x \in D=\left(1,1.074 \times 10^{8}\right), \\
u(x) & =0, \quad x \notin D .
\end{aligned}
$$


i.e.

$$
\begin{aligned}
(A-B \ln x) x u^{\prime}(x)+\frac{1}{2} u^{\prime \prime}(x)+\int_{\mathbb{R} \backslash\{0\}} \frac{u(x+y)-u(x)-y u^{\prime}(x) I_{|y|<1}}{|y|^{1+\alpha}} d y & =-1, \quad x \in D, \\
u(x) & =0, \quad x \notin D .
\end{aligned}
$$

The mean exit time $u(x)$ is the the time when the tumor (initially with density $x$ ) becomes not diagnoseable (when $x(t)$ exits from the left boundary point $x=1$ ), or becomes malignant (when $x(t)$ exits from the right boundary point $x=b$ ) and thus may need medical intervention.

The escape probability $p(x)$, through the right boundary point, is the likelihood that the tumor density $X(t)$, initially at value $x$ in $D$, first escapes from $D$ through $x=1.074 \times 10^{8}$. The $p(x)$ satisfies the following equation Brannan, Duan \& Ervin, 1999]

$$
\begin{aligned}
A p(x) & =0, \quad x \in D=\left(1,1.074 \times 10^{8}\right), \\
p(1) & =0, \quad p\left(1.074 \times 10^{8}\right)=1 .
\end{aligned}
$$

In the next section we simulate both the mean exit time $u(x)$ and escape probability $p(x)$, when the stability parameter $\alpha$ varies in $(0,2)$.

\section{Numerical experiments}

We now discretize the equation (8) for the mean exit time $u(x)$. The discretization of (9) for the escape probability $p(x)$ is similar.

For the numerical scheme as the right boundary point of $D$ is too huge, we need a transformation to change it to a smaller value. Owing to the fact that $u(x)=0$ for $x \notin D$ we can take a transformation $\tilde{x}=\ln x$, relevantly $\tilde{y}=\ln (x+y)-\ln x$, nonetheless the integration for $y$ is on $\mathbb{R} \backslash\{0\}(u(x+y)=0$ for $x+y \leq 0<1)$. Omitting the tilde we get

$$
\begin{aligned}
(A- & B x) u^{\prime}(x)+\frac{1}{2} e^{-2 x}\left(u^{\prime \prime}(x)-u^{\prime}(x)\right) \\
& +\frac{1}{e^{\alpha x}} \int_{\mathbb{R} \backslash\{0\}} \frac{u(x+y)-u(x)-\left(e^{y}-1\right) u^{\prime}(x) I_{\left\{\left|e^{y}-1\right|<e^{-x}\right\}}}{\left|e^{y}-1\right|^{1+\alpha} d y=-1,} \quad x \in D_{1}=(0,18.4921), \\
u(x)=0, \quad x \notin D_{1} . &
\end{aligned}
$$

where the third term in the integral is

$$
\int_{\mathbb{R} \backslash\{0\}} \frac{\left(e^{y}-1\right) I_{\left\{\left|e^{y}-1\right|<e^{-x}\right\}}}{\left|e^{y}-1\right|^{1+\alpha}} e^{y} d y=\int_{\mathbb{R} \backslash\{0\}} \frac{\left(e^{y}-1\right) I_{\left\{\left|e^{y}-1\right|<e^{-x}\right\}}}{\left|e^{y}-1\right|^{1+\alpha}} d\left(e^{y}-1\right)=\int_{\mathbb{R} \backslash\{0\}} \frac{y I_{\left\{|y|<e^{-x}\right\}}}{|y|^{1+\alpha}} d y,
$$

as $\int_{\mathbb{R} \backslash\{0\}} \frac{y I_{\{|y|<\delta\}}}{|y|^{1+\alpha}} d y$ always vanishes for any $\delta>0$, so does the third term in the integral for any fixed $x$, therefore the integral-differential equation can be changed into

$$
\begin{aligned}
& \frac{1}{2} e^{-2 x} u^{\prime \prime}(x)+\left[(A-B x)-\frac{1}{2} e^{-2 x}\right] u^{\prime}(x) \\
&+\frac{1}{e^{\alpha x}} \int_{\mathbb{R} \backslash\{0\}} \frac{u(x+y)-u(x)-\left(e^{y}-1\right) u^{\prime}(x) I_{\left\{\left|e^{y}-1\right|<\delta\right\}}}{\left|e^{y}-1\right|^{1+\alpha} d y}=-1, \quad x \in D_{1}, \\
& u(x)=0, \quad x \notin D_{1} .
\end{aligned}
$$

We then take another transformation $\tilde{x}=\frac{x}{b}-1$ meanwhile $\tilde{y}=\frac{y}{b}$ where $b=18.4921 / 2$ to change $D_{1}=(0,18.4921)$ into $(-1,1)$, we obtain

$$
\begin{array}{r}
\frac{1}{2 b^{2}} e^{-2 b(x+1)} u^{\prime \prime}(x)+\left[A-B b(x+1)-\frac{1}{2} e^{-2 b(x+1)}\right] \frac{1}{b} u^{\prime}(x) \\
+\frac{1}{e^{\alpha b(x+1)}} \int_{\mathbb{R} \backslash\{0\}} \frac{b u(x+y)-b u(x)-\left(e^{b y}-1\right) u^{\prime}(x) I_{\left\{\left|e^{b y}-1\right|<\delta\right\}}}{\left|e^{b y}-1\right|^{1+\alpha}} d y=-1, \quad x \in D_{2}=(-1,1), \\
u(x)=0, \quad x \notin D_{2} .
\end{array}
$$


The numerical scheme here follows [Li, Gao \& Duan, 2011]. A different scheme was also proposed in Chen, Duan, Li \& Zhang, 2011]. We write $\int_{\mathbb{R}}=\int_{-\infty}^{-1-x}+\int_{-1-x}^{1-x}+\int_{1-x}^{\infty}$ and take $\delta=$ $\min \left\{e^{b(1-x)}-1,1-e^{-b(1+x)}\right\}$ to get

$$
\begin{aligned}
& \frac{1}{2 b^{2}} e^{-2 b(x+1)} u^{\prime \prime}(x)+\left[A-B b(x+1)-\frac{1}{2} e^{-2 b(x+1)}\right] \frac{1}{b} u^{\prime}(x) \\
& +\frac{u(x)}{\alpha e^{\alpha b(x+1)}}\left[1-\frac{1}{\left(e^{b(1-x)}-1\right)^{\alpha}}-\frac{1}{\left(1-e^{-b(1+x)}\right)^{\alpha}}\right] \\
& +\frac{1}{e^{\alpha b(x+1)}} \int_{-1-x}^{1-x} \frac{b u(x+y)-b u(x)-\left(e^{b y}-1\right) u^{\prime}(x) I_{\left\{\left|e^{b y}-1\right|<\delta\right\}}}{\left|e^{b y}-1\right|^{1+\alpha}} d y=-1, \quad x \in D_{3}=(-1,1)
\end{aligned}
$$

furthermore, for $x \geq 0$

$$
\begin{aligned}
& \frac{1}{2 b^{2}} e^{-2 b(x+1)} u^{\prime \prime}(x)+\left[A-B b(x+1)-\frac{1}{2} e^{-2 b(x+1)}\right] \frac{1}{b} u^{\prime}(x) \\
& +\frac{u(x)}{\alpha e^{\alpha b(x+1)}}\left[1-\frac{1}{\left(e^{b(1-x)}-1\right)^{\alpha}}-\frac{1}{\left(1-e^{-b(1+x)}\right)^{\alpha}}\right]+\frac{1}{e^{\alpha b(x+1)}} \int_{-1-x}^{-1+x} \frac{u(x+y)-u(x)}{\left|e^{b y}-1\right|^{1+\alpha}} b e^{b y} d y \\
& +\frac{1}{e^{\alpha b(x+1)}} \int_{-1+x}^{1-x} \frac{b u(x+y)-b u(x)-\left(e^{b y}-1\right) u^{\prime}(x)}{\left|e^{b y}-1\right|^{1+\alpha}} e^{b y} d y=-1, \quad x \in D_{3}=(-1,1)
\end{aligned}
$$

and for $x \leq 0$

$$
\begin{aligned}
& \frac{1}{2 b^{2}} e^{-2 b(x+1)} u^{\prime \prime}(x)+\left[A-B b(x+1)-\frac{1}{2} e^{-2 b(x+1)}\right] \frac{1}{b} u^{\prime}(x) \\
& +\frac{u(x)}{\alpha e^{\alpha b(x+1)}}\left[1-\frac{1}{\left(e^{b(1-x)}-1\right)^{\alpha}}-\frac{1}{\left(1-e^{-b(1+x)}\right)^{\alpha}}\right]+\frac{1}{e^{\alpha b(x+1)}} \int_{1+x}^{1-x} \frac{u(x+y)-u(x)}{\left|e^{b y}-1\right|^{1+\alpha}} b e^{b y} d y \\
& +\frac{1}{e^{\alpha b(x+1)}} \int_{-1-x}^{1+x} \frac{b u(x+y)-b u(x)-\left(e^{b y}-1\right) u^{\prime}(x)}{\left|e^{b y}-1\right|^{1+\alpha}} e^{b y} d y=-1, \quad x \in D_{3}=(-1,1)
\end{aligned}
$$

For simplicity denote $f(x)=A-B b(x+1)-\frac{1}{2} e^{-2 b(x+1)}$ and $g(x)=e^{-2 b(x+1)}$. For numerical schemes generally we divide the interval $[-2,2]$ into $4 J$ subintervals and define $x_{j}=j h$ for $-2 J \leq j \leq 2 J$ integer, where $h=1 / J$. We denote the numerical solution of $u$ at $x_{j}$ by $U_{j}$. We can discretize the two integraldifferential equations above using central difference for derivatives and "punched-hole" trapezoidal rule

$$
\begin{aligned}
& \frac{1}{2 b^{2}} g\left(x_{j}\right) \frac{U_{j+1}-2 U_{j}+U_{j-1}}{h^{2}}+\frac{1}{b} f\left(x_{j}\right) \frac{U_{j+1}-U_{j-1}}{2 h} \\
& +\frac{1}{\alpha e^{\alpha b\left(x_{j}+1\right)}}\left[1-\frac{1}{\left(e^{b\left(1-x_{j}\right)}-1\right)^{\alpha}}-\frac{1}{\left(1-e^{-b\left(1+x_{j}\right)}\right)^{\alpha}}\right] U_{j}+\frac{h}{e^{\alpha b\left(x_{j}+1\right)}} \sum_{k=-J-j}^{-J+j} " \frac{b e^{b y_{k}}\left(U_{j+k}-U_{j}\right)}{\left|e^{b y_{k}}-1\right|^{1+\alpha}} \\
& +\frac{h}{e^{\alpha b\left(x_{j}+1\right)}} \sum_{k=-J+j, k \neq 0}^{J-j} " \frac{b e^{b y_{k}}\left(U_{j+k}-U_{j}\right)-e^{b y_{k}}\left(e^{b y_{k}}-1\right)\left(U_{j+1}-U_{j-1}\right) / 2 h}{\left|e^{b y_{k}}-1\right|^{1+\alpha}}=-1,
\end{aligned}
$$

where $j=0,1,2, \cdots, J-1$. Here the summation symbol $\sum^{\prime \prime}$ means the two end terms are multiplied by $1 / 2$ corresponding to trapezoidformula.

$$
\begin{aligned}
& \frac{1}{2 b^{2}} g\left(x_{j}\right) \frac{U_{j+1}-2 U_{j}+U_{j-1}}{h^{2}}+\frac{1}{b} f\left(x_{j}\right) \frac{U_{j+1}-U_{j-1}}{2 h} \\
& +\frac{1}{\alpha e^{\alpha b\left(x_{j}+1\right)}}\left[1-\frac{1}{\left(e^{b\left(1-x_{j}\right)}-1\right)^{\alpha}}-\frac{1}{\left(1-e^{-b\left(1+x_{j}\right)}\right)^{\alpha}}\right] U_{j}+\frac{h}{e^{\alpha b\left(x_{j}+1\right)}} \sum_{k=J+j}^{J-j} " \frac{b e^{b y_{k}}\left(U_{j+k}-U_{j}\right)}{\left|e^{b y_{k}}-1\right|^{1+\alpha}} \\
& +\frac{h}{e^{\alpha b\left(x_{j}+1\right)}} \sum_{k=J+j, k \neq 0}^{-J-j} " \frac{b e^{b y_{k}}\left(U_{j+k}-U_{j}\right)-e^{b y_{k}}\left(e^{b y_{k}}-1\right)\left(U_{j+1}-U_{j-1}\right) / 2 h}{\left|e^{b y_{k}}-1\right|^{1+\alpha}}=-1,
\end{aligned}
$$


where $j=-(J-1),-(J-2), \cdots,-2,-1$. The boundary conditions require that the values of $U_{j}$ vanish if the index $|j| \geq J$. But here for the specific problem for first-order derivative we use forward difference or backward difference.

Similarly, we can discretize the equation (9) for the escape probability $p(x)$.

For the viewing convenience, the following figures for the mean exit time $u(x)$ and escape probability $p(x)$ are both plotted for $x \in(0,18.4921)$, instead of the original huge range $\left(1,1.074 \times 10^{8}\right)$.

Figures 16 show the mean exit time $u(x)$ for various $\alpha$ values in $(0,2)$.

We observe that when $\alpha$ is small, the mean exit time $u(x)$ is small and is roughly constant, i.e., tumors at all densities are equally unlikely to become non-diagnosable or malignant. As $\alpha$ becomes bigger the whole height will grow with the right part grows up slowly while the left part increases rapidly meanwhile the width of the left part narrows down. In fact, at about $\alpha=0.2$ the height of the two part begins to have a separation. When $\alpha \approx 0.44$ the separation becomes quite obvious and the left part (for density between 0 and 10) reaches its highest value, which indicates a bifurcation. This means that for a tumor with density between 0 and 10, it takes longest time to become either non-diagnosable or malignant.

For $\alpha$ between 0.44 and $0.48, u(x)$ keeps similar shape as for $\alpha=0.44$ but the peak diminishes. For $\alpha$ between 0.48 and $0.6, u(x)$ has the similar shape although the peak value decreases. Moreover, for $\alpha$ between 0.6 and 2, $u(x)$ keeps almost the same shape; namely, for the Gaussian Brownian motion case, the mean exit time is similar to the large values of $\alpha \in(0.6,2)$. The dramatic impact of non-Gaussian Lévy noise occurs for $\alpha \in(0,0.46)$.

Figure 7 shows a three dimensional plot of the above discussed situations.

In the above simulations, the mean exit time $u(x)$ tells us the time for a tumor to become either nondiagnosable (exit from the left boundary point) or become malignant (exit from the right boundary point). To distinguish these two situations, especially examine the situation for a tumor to become malignant, let us now consider the escape probability, $p(x)$, through the right end point. It indicates the likelihood the the tumor is progressing from benign to malignant. We observe that a bifurcation occurs for $\alpha \approx 0.4$ when a tumor at any benign density will highly likely becomes malignant, while for $\alpha<0.4$, only tumors with high density (near $x=20$ ) are likely to become malignant; see Figures 8 and 9 .

Acknowledgements. We thank Xiaofan Li and Ting Gao for help with numerical schemes, and Xu Sun for helpful discussions on the model.

\section{References}

Albano, G. \& Giorno, V. [2006] "A stochastic model in tumor growth," Journal of Theoretical Biology. 242, pp. 329-336.

Albano, G. \& Giorno, V. [2009] "On the First Exit Time Problem for a Gompertz-Type Tumor Growth," Lecture Notes in Computer Science, 5717, pp. 113-120.

Albeverrio, S., Rüdiger, B. \& Wu, J. L. [2000] "Invariant Measures and Symmetry Property of Lévy Type Operators," Potential Analysis, 13, pp. 147-168.

Applebaum, D. [2009] Lévy Processes and Stochastic Calculus, 2nd ed, Cambridge University Press, Cambridge, UK.

Biagini, F., Hu, Y., Øksendal, B. \& Zhang, T. [2006] Stochastic Calculus for Fractional Brownian Motion and Application, Springer.

Brannan, J., Duan, J. \& Ervin, V. [1999] "Escape Probability, Mean Residence Time and Geophysical Fluid Particle Dynamics," Physica D, 133, pp. 23-33.

Caffarelli, L. \& Silvestre, L. [2007] "An Extension Problem Related to the Fractional Laplacian," Communications in Partial Differential Equations.

Chen, H., Duan, J., Li, X. \& Zhang, C. [2011] "A computational analysis for mean exit time under nonGaussian Lévy nioses," Applied Math and Comput.

Chen, Z. Q., Kim, P. \& Song, R. [2010] "Heat kernel estimates for Dirichlet fractional Laplacian," J. European Math. Soc. 12, pp. 1307-1329. 
Feller, W. [1971] An introduction to Probability Theory and its Application, Vol.2, 2nd Ed. Wiley.

Guan, Q. Y. \& Ma, Z.-M. [2005] "Boundary Problems for Fractional Laplacians," Stochastics and Dynamics, 5, p. 385.

Gutiérrez-jáimez, R., Román, P., Romero, D., Serrano, J. \& Torres, F. [2007] "A new Gompertz-type diffusion process with application to random growth," Mathematical Biosciences 208, pp. 147-165.

Kyprianou, A. E. [2006] Introductory Lectures on Fluctuations of Lévy Processes with applications, Springer. Li, X., Gao, T. \& Duan, J. [2011] "Mean exit time and escape probability for dynamical systems driven by non-Gaussian noise," Submitted.

Lo, C. F. [2007] "Stochastic Gompertz model of tumor cell growth," Journal of Theoretical Biology. 248, pp. 317-321.

Lo, C. F. [2009] "Stochastic Nonlinear Gompertz Model of Tumor Growth," Proceedings of the World Congress on Engineering.

Parfitt, A. M. \& Fyhrie, D. P. [2009] "Gompertzian growth curves in parathyroid tumors: further evidence for the set-point hypothesis," Cell Prolif. 30, pp. 341-349.

de Vladar, H. P. \& Gonzalez, J. A. [2004] "Dynamic response of cancer under the influence of immunological activity and therapy," Journal of Theoretical Biology. 227, pp. 335-348. 

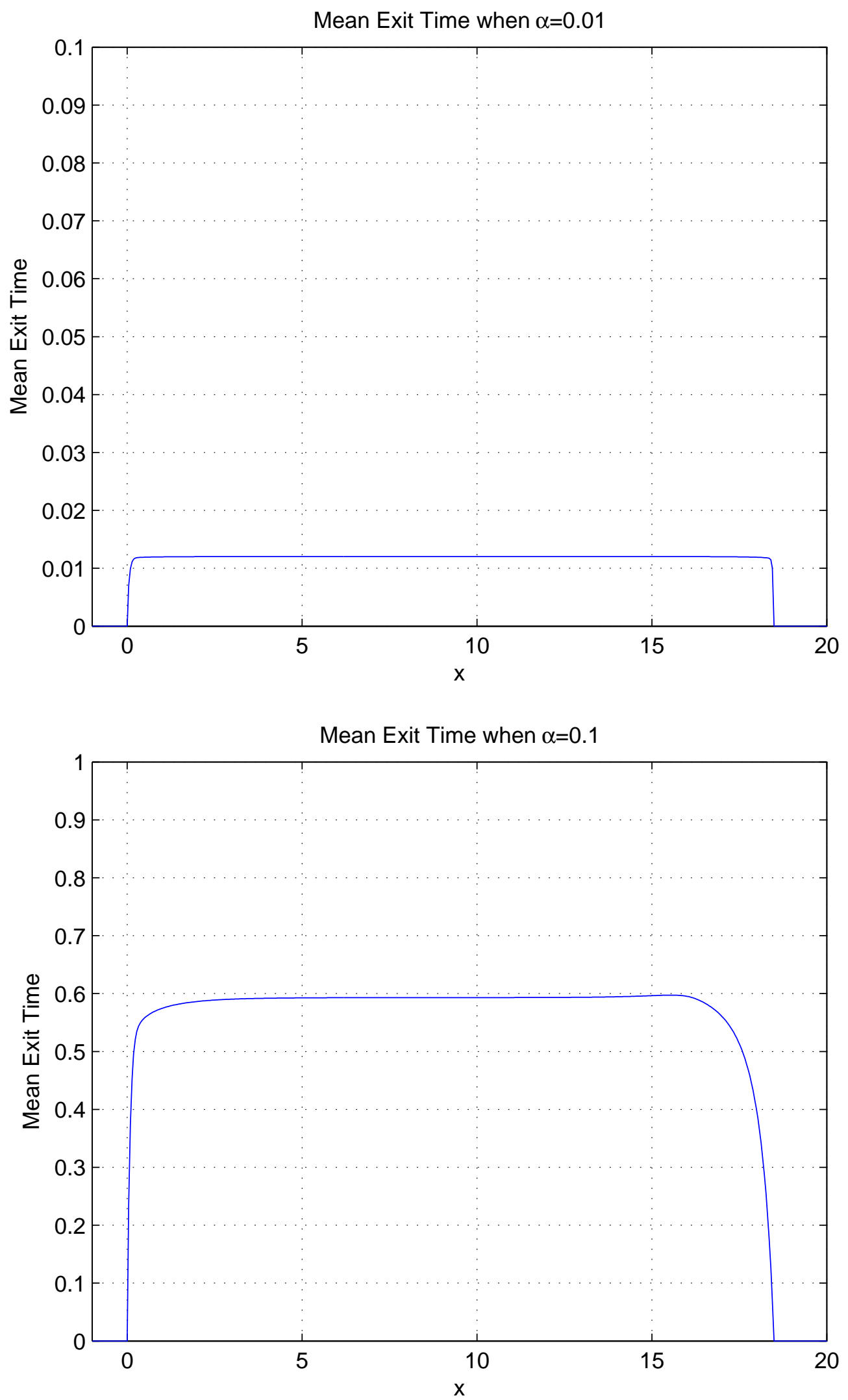

Fig. 1. Plot of $u(x)$ given by (10) with $\alpha=0.01$ and $\alpha=0.1$. 

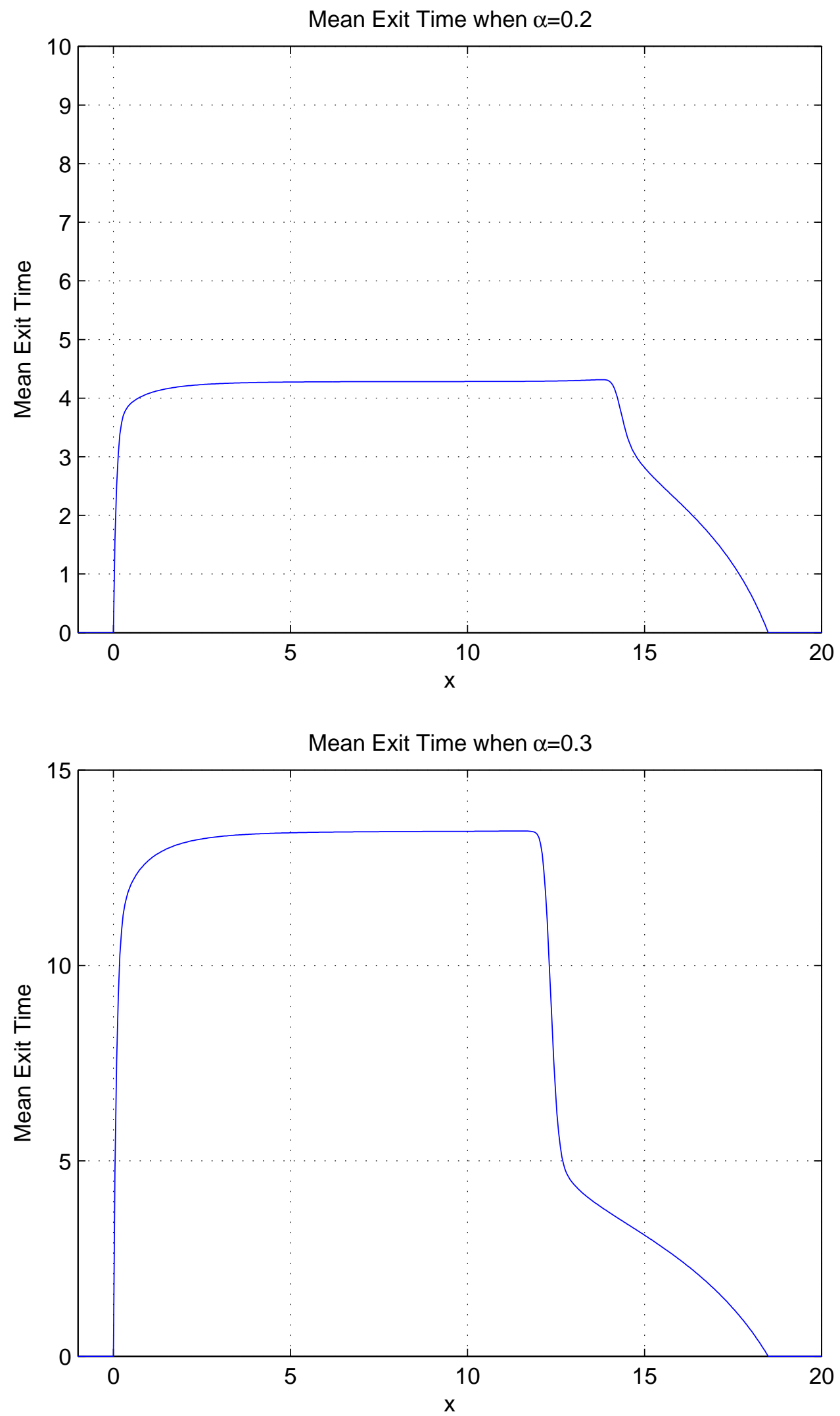

Fig. 2. Plot of $u(x)$ given by (10) with $\alpha=0.2$ and $\alpha=0.3$. 

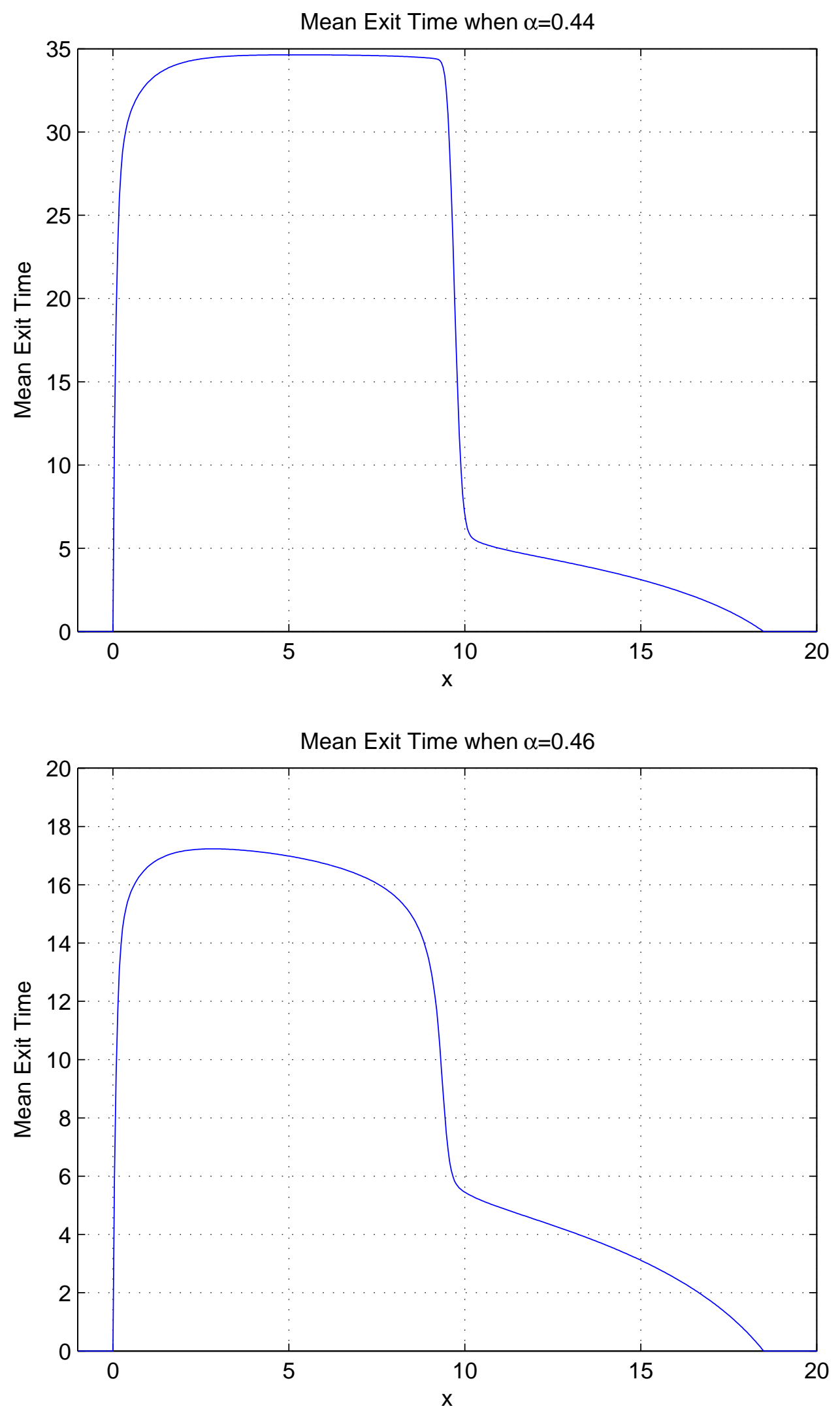

Fig. 3. Plot of $u(x)$ given by (10) with $\alpha=0.44$ and $\alpha=0.46$. 

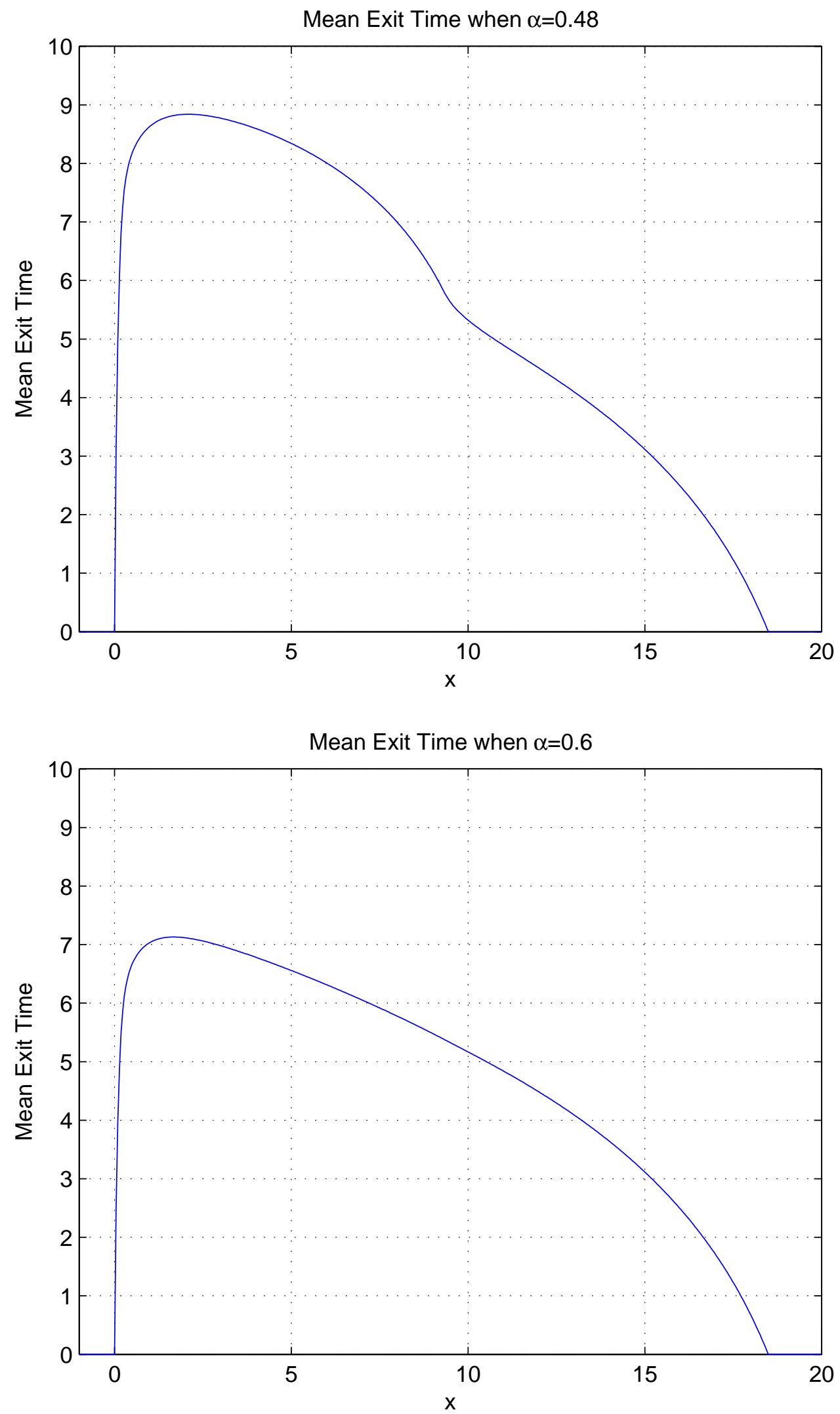

Fig. 4. Plot of $u(x)$ given by (10) with $\alpha=0.48$ and $\alpha=0.6$. 

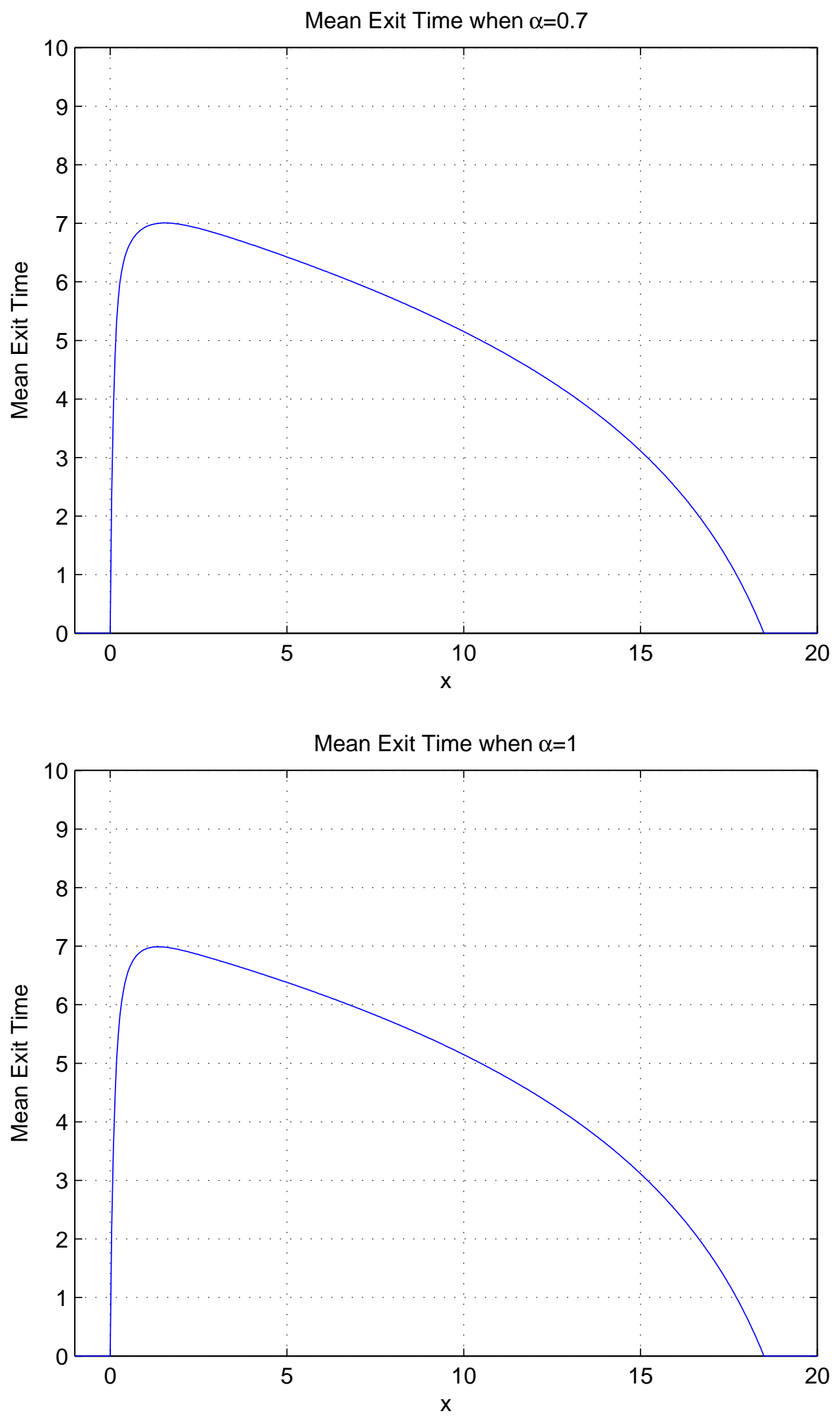

Fig. 5. Plot of $u(x, t)$ given by (10) with $\alpha=0.7$ and $\alpha=1$. 

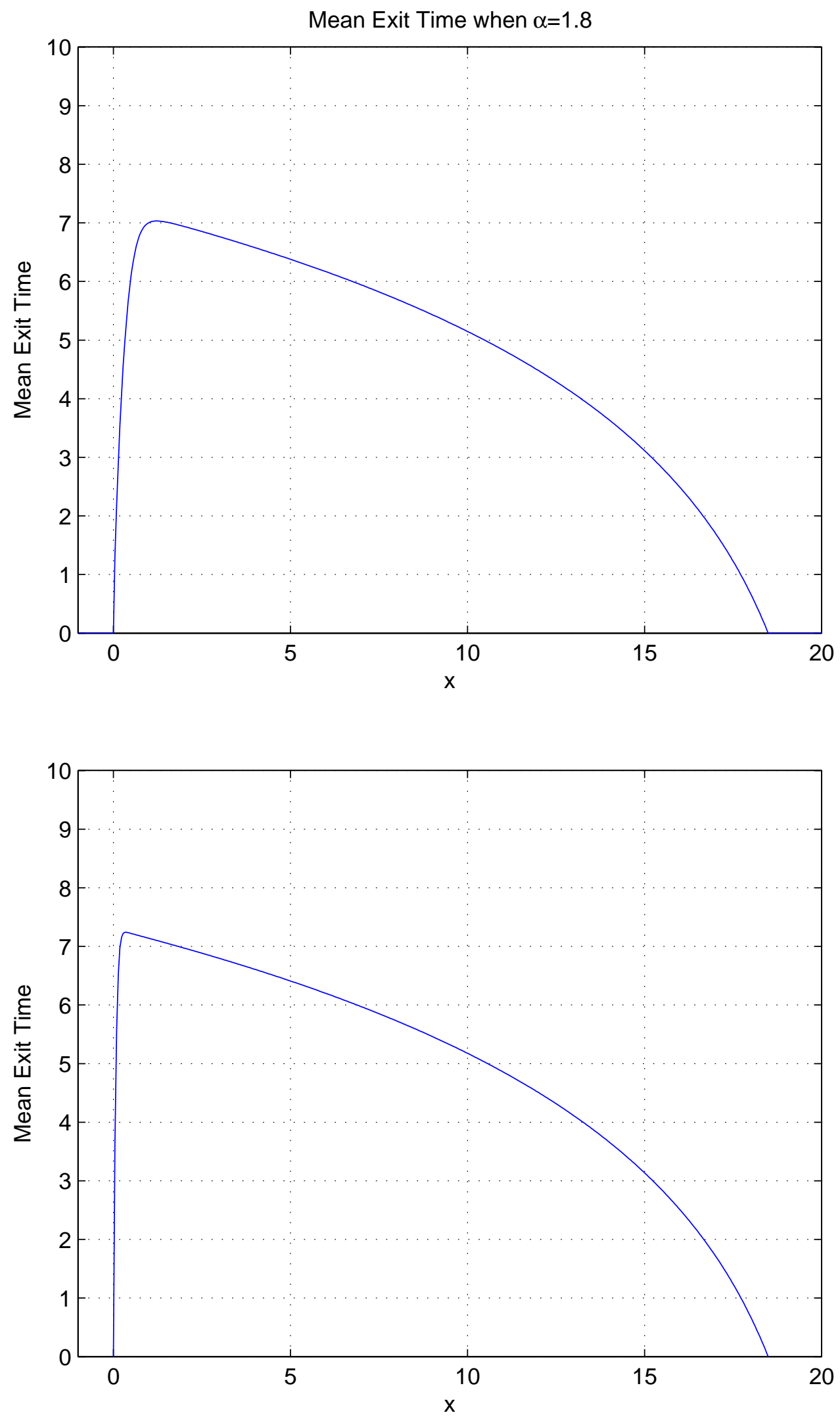

Fig. 6. Plot of $u(x, t)$ given by (10) with $\alpha=1.8$ and the Brownian motion case. 


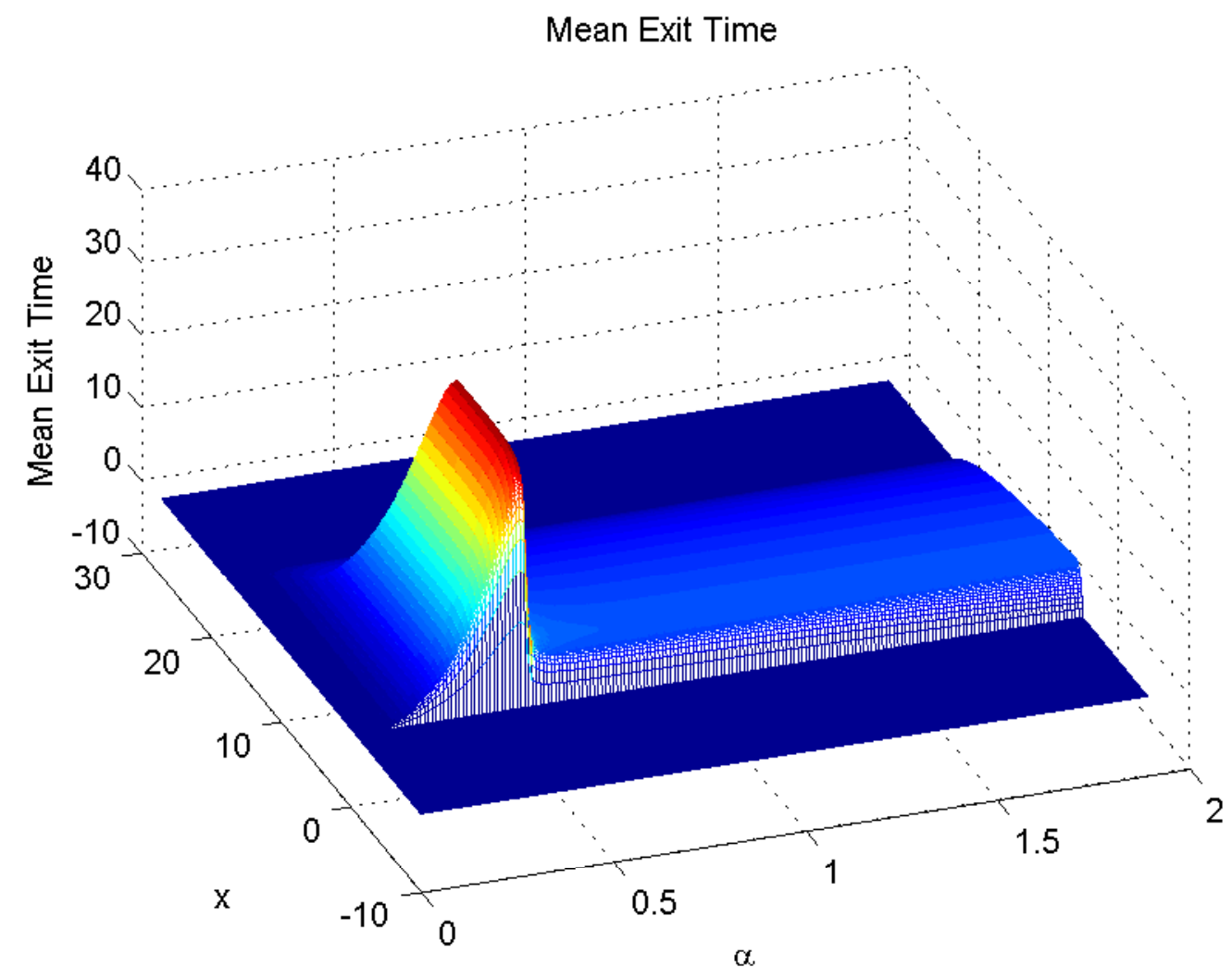

Fig. 7. Mean exit time $u$ v.s. $\alpha$ and $x$. 

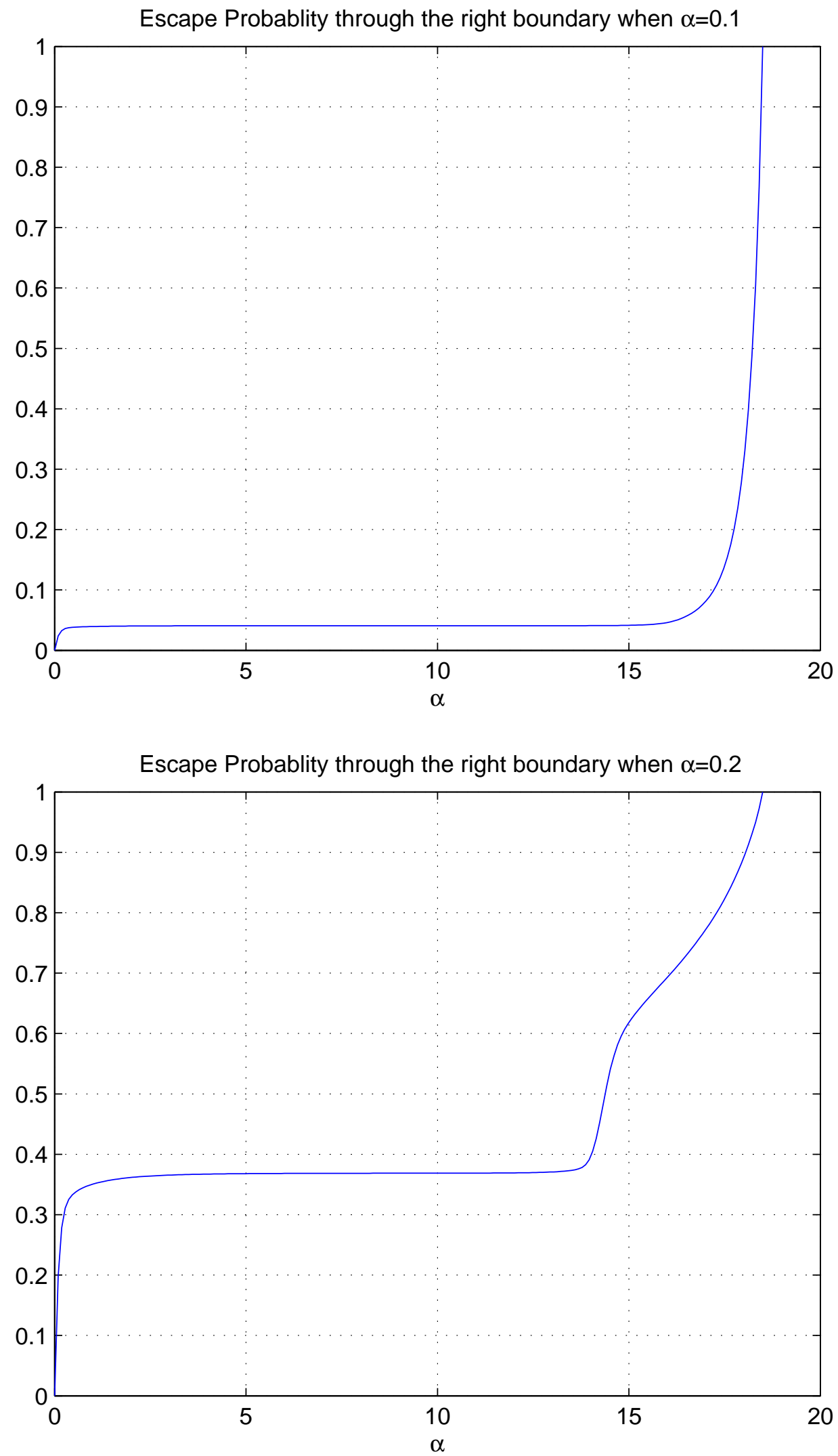

Fig. 8. Escape probability $p(x)$ through the right boundary of $(0,18.4921): \alpha=0.1$ and $\alpha=0.2$. 


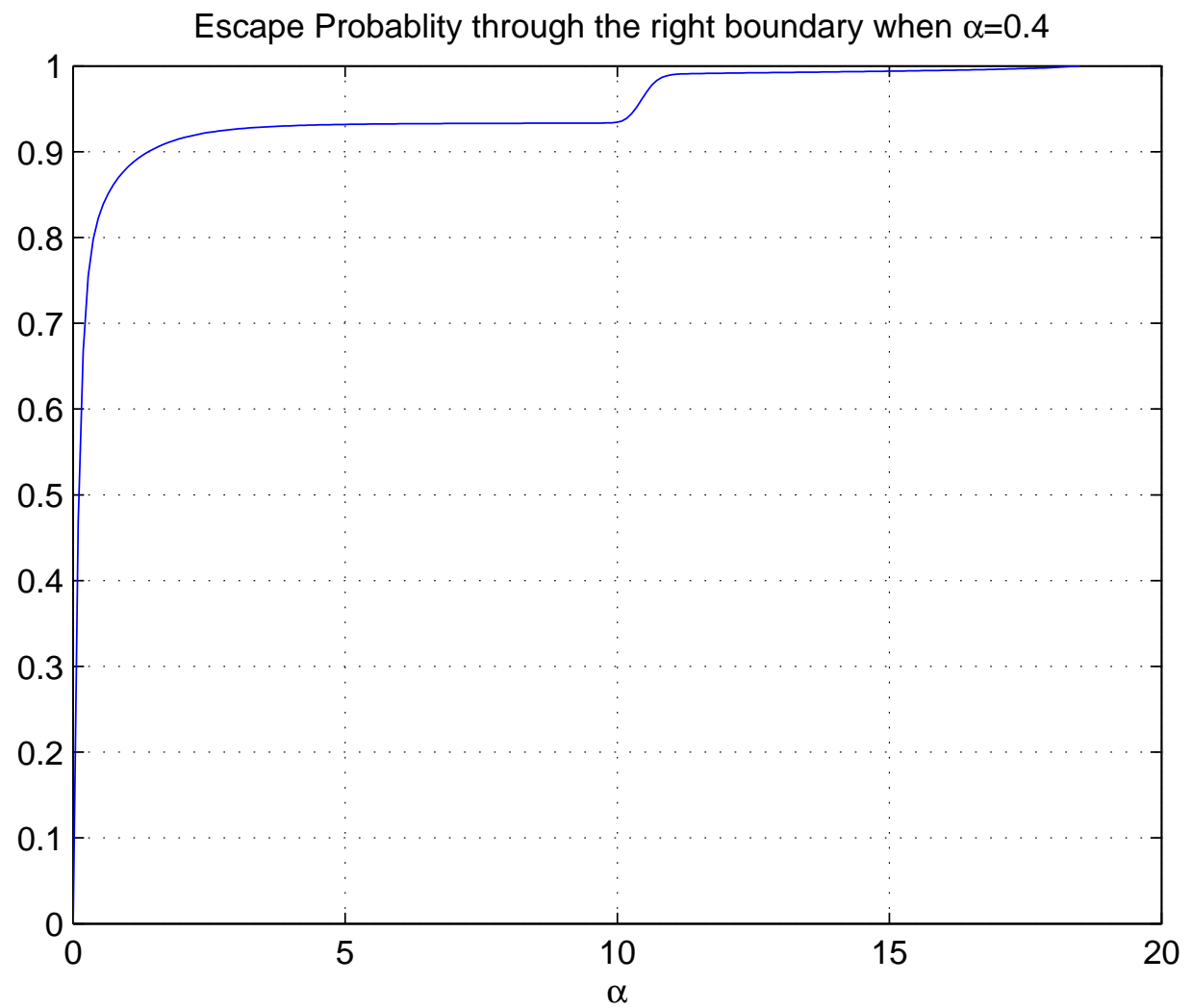

Escape Probablity through the right boundary when $\alpha=0.5$

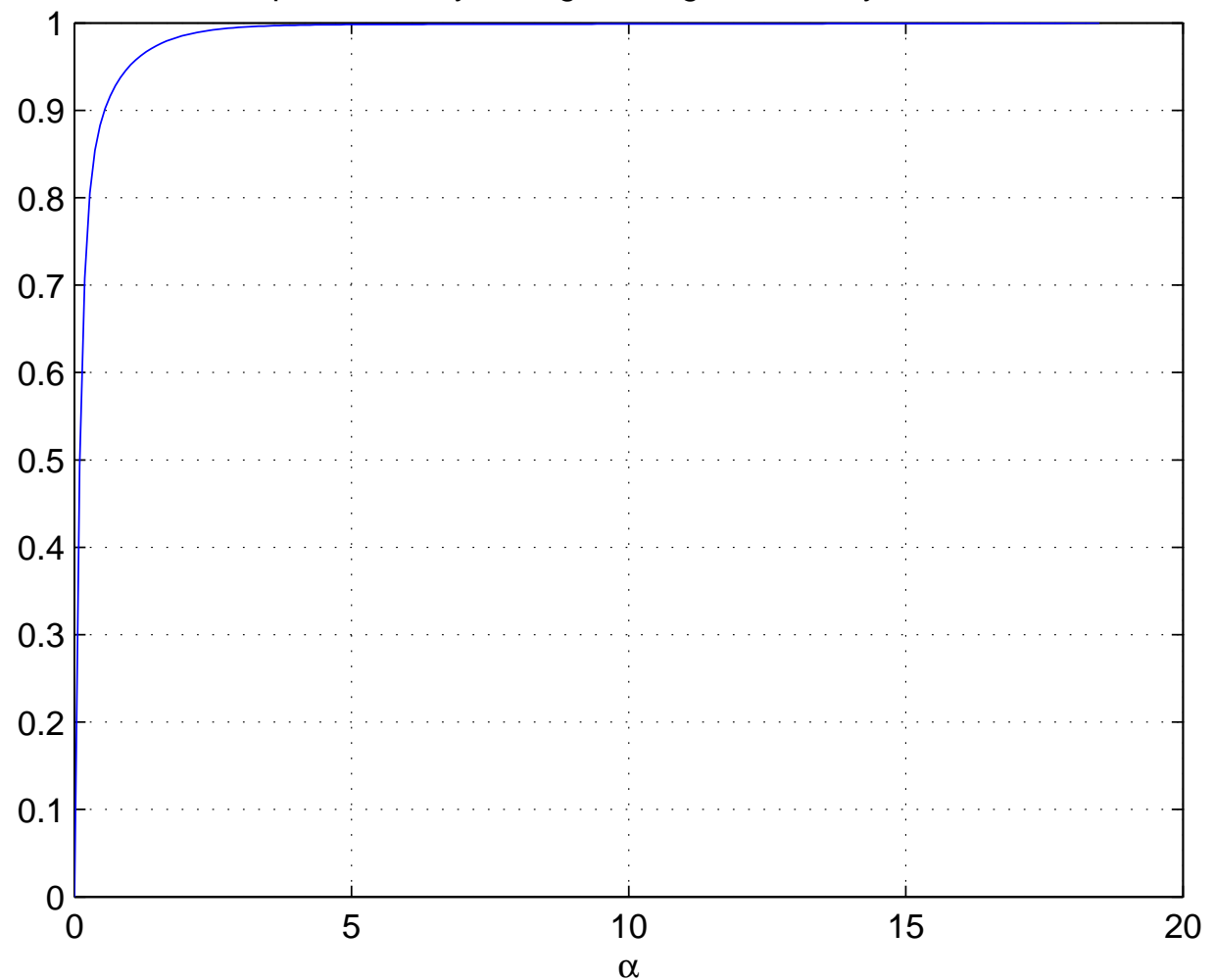

Fig. 9. Escape probability $p(x)$ through the right boundary of $(0,18.4921): \alpha=0.4$ and $\alpha=0.5$. 